\title{
The correlation between the health-related fitness of healthy participants measured at home as opposed to fitness measured by sport scientists in a laboratory
}

\author{
CC Grant ${ }^{a *}$, DC Janse van Rensburga, MS Pepperb, PJ du Toitc, PS Wood d, J Kera , PE Krügere, CW Grobbelaarc, K Nolte ${ }^{d}$, \\ F Fletcherf and TC Grant ${ }^{\mathrm{a}}$
}

\author{
${ }^{a}$ Department of Sports Medicine, University of Pretoria, Pretoria, South Africa \\ ${ }^{b}$ Department of Immunology, University of Pretoria, Pretoria, South Africa \\ 'Department of Physiology, University of Pretoria, Pretoria, South Africa \\ ${ }^{d}$ Department of Biokinetics, Sport and Leisure Sciences, University of Pretoria, Pretoria, South Africa \\ ${ }^{e}$ Institute for Sport Research, University of Pretoria, Pretoria, South Africa \\ ${ }^{f}$ Department of Statistics, University of Pretoria, Pretoria, South Africa \\ *Corresponding author, email:rina.grant@up.ac.za
}

\begin{abstract}
Background: Fitness is defined in the health context as a state of good health or physical condition, primarily as a result of exercise and proper nutrition. Conventional methods of measuring fitness are expensive, time consuming and require specialised methods. There is a need for noninvasive, fast methods of assessing health-related fitness and activity in athlete and non-athlete populations. The aim of this study was to establish any correlation between participants'self-assessed health-related fitness (HRF) index and the HRF index determined by sports scientists, as well as the laboratory-assessed HRF index and maximum oxygen uptake $\left(\mathrm{VO}_{2} \mathrm{max}\right)$ determined by sports scientists via direct methods in a laboratory, and finally, to determine any potential correlation between an activity-recall questionnaire (Kasari) and $\mathrm{VO}_{2}$ max.

Method: The participants consisted of 169 male and female volunteers between the ages of 18 and 55 years, taking neither supplements nor medication. The University of Pretoria's HRF index protocol was first carried out by participants on their own at home, and then in the laboratory under the supervision of sport scientists. The complete datasets of 150 participants were statistically analysed to establish correlations.

Results: The results indicated highly significant and substantial correlations $(\rho=0.696, p<0.001)$ between the participants' self-assessed HRF index and measurements by the sports scientists. There were moderate correlations between the laboratoryassessed HRF index and the direct $\mathrm{VO}_{2} \max (\rho=0512, p<0.005)$, as well as the indirect $\mathrm{VO}_{2} \max (\rho=0.588 ; p<0.001)$. A significant correlation was found between the self-assessed HRF index and the indirect $\mathrm{VO}_{2} \max (\rho=0.454 ; p<0.001)$. The Kasari questionnaire correlated only weakly with the indirect $\mathrm{VO}_{2} \max (\rho=0.278, p<0.002)$ and not at all with the direct $\mathrm{VO}_{2} \max$ $(\rho=0.072, p=0.731)$.

Conclusion: Indications are that this test protocol may be conducted at home by individuals, without supervision, to determine exercise ability or fitness, or to monitor changes in physical fitness, thereby limiting expenses and costs.
\end{abstract}

Keywords: noninvasive measurements, physical fitness, pedometer, $\mathrm{VO}_{2}$ max

\section{Introduction}

Fitness is defined as a state of good health or physical condition, primarily as a result of exercise and proper nutrition. Controversy has arisen as to what the components of physical fitness are, and this led to the grouping of physical fitness into health-related fitness and skill-related physical fitness. ${ }^{1}$ Health-related fitness focuses mainly on the optimum health of the individual. The five major components of health-related fitness are cardiorespiratory endurance, body composition, muscular strength, muscular endurance and flexibility. ${ }^{1,2}$

Maximum oxygen uptake ( $\left.\mathrm{VO}_{2} \mathrm{max}\right)$, the maximum rate at which oxygen can be consumed, is a commonly used measure of cardiorespiratory (or aerobic) fitness. ${ }^{3}$ It is also called maximal oxygen uptake or aerobic capacity, and indicates the maximum capacity of an individual's body to transport and utilise oxygen during incremental exercise. $\mathrm{VO}_{2}$ max is expressed in terms of millilitres of oxygen consumed per kilogram of body weight per minute. Various predictive tests have been devised to evaluate aerobic fitness, which include performance-related measures, such as the Submaximal Cycle Ergometer test, multistage progressive shuttle test and the Cooper 12 minute walk/run test. ${ }^{3}$ The latter has been reported to be the best predictor of $\mathrm{VO}_{2} \mathrm{max}^{3}$

$\mathrm{VO}_{2} \max$ is also known to be a strong and independent predictor of cardiovascular disease and mortality, providing reproducible data. A longitudinal study by Paterson et al. indicated that $\mathrm{VO}_{2} \mathrm{max}$ is one of only three factors, excluding age and comorbidity, able to predict future dependence in the elderly. ${ }^{4}$ $\mathrm{VO}_{2}$ max has been widely used as a predictor of survival and performance in a variety of clinical and athletic populations. ${ }^{4,5}$

Conventional methods of measuring physical fitness, such as $\mathrm{VO}_{2}$ max, are expensive, time consuming and require specialised methods. Although many studies use a memory-based questionnaire as an alternative indicator of fitness, recall bias is a known complication. ${ }^{6}$ Thus, there is a need for a noninvasive, fast method of assessing health-related fitness in athlete and nonathlete populations, as well as in patient groups.

The University of Pretoria health-related fitness index (HRF index), developed by the university, may be used as an indicator 
of exercise capacity and activity. It calculates a fitness index, based on the most important components of health-related fitness, and can be used by both athlete and clinical populations. It consists of a battery of 10 tests which can easily be performed by participants at home under self-supervision, and in the laboratory under standardised conditions.

The purpose of this study was to establish a correlation between the University of Pretoria HRF index of participants measured at home by themselves, the HRF index of participants measured under standardised laboratory conditions, the direct or indirect $\mathrm{VO}_{2}$ max and a memory-based questionnaire, Kasari, on activity. It was hypothesised that there are significant correlations between:

- The participants' self-assessed HRF index and the HRF index determined by sports scientists.

- The laboratory-assessed HRF index and the $\mathrm{VO}_{2}$ max, determined by sports scientists via direct methods in a laboratory.

- An activity-recall questionnaire (Kasari) and $\mathrm{VO}_{2}$ max.

\section{Method}

Before the onset of the project, a research protocol was submitted and approved by the Research Ethics Committee of the Faculty of Health Sciences at the University of Pretoria. An invitation via e-mail was sent to University of Pretoria students and staff to participate in the research project. Inclusion criteria included healthy male and females between the ages of 18 and 55 years of age. Activity status was noted, but not used as an inclusion or exclusion criterion. Participation exclusion criteria included participants who refused to provide informed consent, those with a history of cardiovascular disease, endocrine or metabolic disorders, those with a lung or respiratory tract infection in the previous two weeks, and those who were on medication that could influence cardiovascular control. A pre-study orientation session was held, whereby participants were screened to ensure compliance with the criteria for participation.

\section{Participants}

The volunteers consisted of 169 male and females between the ages of 18 and 55 years (Table 1). Participants were informed of the benefits and risks associated with the study. Signed consent forms were obtained from volunteers prior to the study. Only participants with complete datasets $(n=150)$ were included in the data analyses.

\section{The health-related fitness index}

The HRF index protocol consisted of the following 10 tests:

Body composition:

- Body mass index (BMI).

- Waist-to-hip ratio.

- Waist circumference (abdominal fat).

\section{Cardiorespiratory fitness:}

- Twelve minute walk/run test to calculate a predicted maximal oxygen consumption test $\left(\mathrm{VO}_{2} \mathrm{max} / \mathrm{VO}_{2}\right.$ peak).

Table 1: Descriptive statistics of the participants. Mean and standard deviation (SD) are shown

\begin{tabular}{lcc}
\hline Descriptive statistics & Male & Female \\
\hline$n$ & 43 & 126 \\
Weight & \multicolumn{2}{c}{68.83 (SD 14.32) } \\
Body mass index & \multicolumn{2}{c}{23.83 (SD 3.98) } \\
Age & \multicolumn{2}{c}{21.96 (SD 4.64) } \\
\hline
\end{tabular}

Muscular strength and endurance:

- One-minute push-ups.

- One-minute, bent-knee sit-ups.

- Leg-bend test.

Flexibility:

- Sit and reach test.

- Shoulder flexibility test.

Balance:

- Stork stand test.

BMI was calculated using the following formula: body weight $(\mathrm{kg}) /$ height squared $\left(\mathrm{m}^{2}\right)=\mathrm{BMI}\left(\mathrm{kg} / \mathrm{m}^{2}\right)$. The waist-to-hip ratio is the waist measurement $(\mathrm{mm})$ divided by largest hip circumference $(\mathrm{mm})$.

\section{Indirect $\mathrm{VO}_{2} \max (\mathrm{ml} / \mathrm{kg} /$ minute) as calculated from the Cooper 12 minute walk/run test}

Using the 12 minute walk/run test, participants had to walk or run continuously for 12 minutes. The aim was to cover as much distance as possible. For the one-minute push-ups and one-minute, bent-knee sit-ups, participants had to accomplish as many sit-ups or push-ups as possible in a one-minute period. For the leg-bend test, participants had to stand on one leg and bend that leg until they could not see a line right in front of that foot. They then determined the average amount of leg bends that they could do in 30 seconds using both legs. The sit and reach test determined how far they could stretch with their hand while sitting flat on the floor, legs extended in front of them and reaching towards their feet. With the shoulder flexibility test, participants had to stand straight, place one arm behind the other, with fingers pointing down between the shoulder blades and the palm lying flat against the back. They then brought the other hand up behind the back, palm outward as far as it would go, without undue stain. With the stork stand test, participants had to remove their shoes and stand up straight with their hands on their hips. They then placed the left foot against the inside knee of the right leg. Then, raising the heel of the right leg, they stood on the ball of the foot only for as long as possible.

These tests were first carried out by participants on their own at home, and then in the laboratory under the supervision of biokineticists and sport scientists from the Institute for Sport Research, University of Pretoria.

\section{$\mathrm{VO}_{2} \max$}

Indirect measurement of the $\mathrm{VO}_{2}$ max was performed on the whole group $(n=169)$ and direct $\mathrm{VO}_{2}$ max was included for some of the participants $(n=29)$. This cardiopulmonary exercise test provides diagnostic and prognostic clinical information and evaluates cardiorespiratory fitness. $\mathrm{VO}_{2}$ max is normally assessed in a laboratory setting using respiratory gas analysis equipment. This direct determination of $\mathrm{VO}_{2}$ max is the preferred method of assessing aerobic capacity. ${ }^{5}$ However, because of the large number of participants in the current study, direct determination of aerobic capacity was only performed in a subgroup of participants.

The direct $\mathrm{VO}_{2}$ max exercise test consisted of a continuous progressive treadmill exercise test to volitional exhaustion. Participants were permitted five minutes of light stretching prior to a five-minute run at approximately $10 \mathrm{~km} /$ hour-1 to warm up. Thereafter, treadmill speed was increased by $1 \mathrm{~km} /$ hour-1 every 
minute and grade by $0.5 \%$ every two minutes. Pulmonary gas exchange and heart rate were analysed continuously during the test using an automated ergospirometer and electrocardiograph $\left(\mathrm{Cosmed}^{\circ} \mathrm{K} \mathrm{b}^{2}\right)$. $\mathrm{VO}_{2}$ max was recorded as the highest oxygen uptake $\left(\mathrm{VO}_{2}\right)$ averaged over 30 seconds of the test and reported in relative terms ( $\mathrm{ml} / \mathrm{kg}$ body mass/minute).

The whole group $(n=150)$ completed the Cooper 12 minute walk/run test. This assessment uses a set period ( 12 minutes) and scoring according to distance (in metres), rather than marking a set distance and using time as the assessment. This test was performed on a $400 \mathrm{~m}$ tartan athletics track. A warm up of light aerobic activities and flexibility exercises was performed for five minutes before the start of the test. Testers were placed at $100 \mathrm{~m}$ intervals around the track as a source of verbal motivation. A prediction of $\mathrm{VO}_{2}$ max from the distance covered at the end of the 12 minute period was obtained by applying the distance run to the Cooper regression equation: $\mathrm{VO}_{2} \max (\mathrm{ml} / \mathrm{kg} / \mathrm{minute})=0.0268$ (distance covered in metres) $-11.3^{7}$

\section{Kasari index}

Activity was determined with the aid of the validated Kasari physical activity questionnaire. It was developed in the 1970s by Kasari, and was shown to relate to aerobic fitness, i.e. with an increase in the frequency, intensity, and time (FIT) of exercise, there is an accompanying increase in the index score and fitness. ${ }^{8}$ The FIT index of Kasari quantifies respondents' participation in any form of leisure time physical activity. The questionnaire uses three parameters, namely frequency of exercise, intensity of exercise and time spent on workout, to calculate the FIT index using the following formula: FIT index = (points for frequency) $\mathrm{x}$ (points for intensity) $\mathrm{x}$ (points for duration). A minimum score of 1 and a maximum score of 100 can be achieved. A higher score is indicative of a more physically active person. The FIT index of Kasari has been successfully used in South African studies. ${ }^{9,10}$

\section{Data analysis}

The study was conducted according to the protocol and in compliance with the guidelines for good clinical practice. The information obtained from the participants was captured on computer and analysed by means of the IBM SPSS ${ }^{\circ}$ Statistics version 19 package.

Owing to the skewed nature of the variables, Spearman's rank correlation coefficient $(\rho)$, which is a nonparametric measure of strength and direction of statistical dependence, was calculated (instead of Pearson's product-moment correlation coefficient $r$ ). Spearman's $\rho$ assesses how well the relationship between two variables can be described using a monotonic function, i.e. $\rho$ indicates concordance or discordance. A coefficient of 0 means that the variables are not monotonically related. ${ }^{11}$ The advantages of this measure are that the variables do not need to be normally distributed and it is not very sensitive to outliers.

The usual $5 \%$ level of significance $(\alpha=0.05)$ was used to test whether $\rho$ differed significantly from zero, i.e. to determine if there was a statistically significant monotonic relationship between the pairs of variables. However, correlations with $p$ larger than 0.05 , but less than 0.1 , are also reported as indicative of moderate evidence of a relationship.

\section{Results}

The descriptive statistics of the participants and tests are listed in Tables 1 and 2, respectively.
Table 2: Descriptive statistics of the tests

\begin{tabular}{|c|c|c|c|c|}
\hline Descriptive statistics & Minimum & Maximum & Mean & SD \\
\hline Self-assessed HRF index & 15.00 & 29.00 & 22.42 & 3.17 \\
\hline $\begin{array}{l}\text { Laboratory-assessed HRF } \\
\text { index }\end{array}$ & 14.00 & 28.00 & 22.68 & 2.87 \\
\hline $\begin{array}{l}\text { Indirect } \mathrm{VO}_{2} \max (\mathrm{ml} / \mathrm{kg} / \\
\text { minute), calculated from } \\
\text { the Cooper } 12 \text { minute } \\
\text { walk/run test }\end{array}$ & 17.42 & 72.68 & 40.11 & 10.38 \\
\hline $\begin{array}{l}\text { Direct } \mathrm{VO}_{2} \max (\mathrm{ml} / \mathrm{kg} / \\
\text { minute) }\end{array}$ & 31.90 & 69.71 & 47.60 & 8.44 \\
\hline Kasari index & 4.00 & 14.00 & 10.75 & 1.77 \\
\hline
\end{tabular}

HRF: health-related fitness, SD: standard deviation, $\mathrm{VO}_{2}$ max: maximum oxygen uptake

Table 3: Landis and Koch classification scale

\begin{tabular}{ll}
\hline Association value & Interpretation \\
\hline 0.00 & Poor \\
$0.01-0.20$ & Slight \\
$0.21-0.40$ & Fair \\
$0.41-0.60$ & Moderate \\
$0.61-0.80$ & Substantial \\
$0.81-1.00$ & Almost perfect \\
\hline
\end{tabular}

The Landis and Koch reliability classification scale, shown in Table 3, was used to label the strength of the correlation statistics with adjectives. ${ }^{12}$

The results in Table 4 indicate highly significant and substantial positive correlations $(\rho=0.696, p<0.001)$ between the participants' self-assessed HRF index and the measurements by biokineticists and sports scientists. There was moderate correlation between the laboratory-assessed HRF index and the direct $\mathrm{VO}_{2} \max (\rho=0.512$, $p=0.005)$, as well as the indirect $\mathrm{VO}_{2} \max (\rho=0.588, p<0.001)$. The Kasari questionnaire correlated only weakly with the indirect $\mathrm{VO}_{2} \max (\rho=0278, p=0.002)$, and not at all with the direct $\mathrm{VO}_{2}$ max $(\rho=0.072, p=0.731)$. Although the participants' self-assessed HRF index did not correlate significantly with the direct $\mathrm{VO}_{2}$ max ( $\rho=0.387, p=0.068$ ), a highly significant correlation was found between the self-assessed HRF index and the indirect $\mathrm{VO}_{2}$ max $(\rho=0.454, p<0.001)$.

\section{Discussion}

The University of Pretoria HRF index consisted of a report of physical fitness based on physical tests performed by the participants. This was measured and reported firstly by the participant themselves, and secondly by biokineticists and sport scientists. Highly significant correlations were found between these measurements. However, only a weak correlation was found between the Kasari questionnaire and the indirect $\mathrm{VO}_{2}$ max ( $\rho=0.278, p=0.002$ ), as measured by the Cooper 12 minute walk/ run test. This shows that the Kasari physical activity questionnaire was not a good indicator of participants' fitness and is therefore not a reliable substitute for cardiorespiratory fitness testing.

The accurate quantification of physical activity and physical fitness are essential in terms of health outcome and the effectiveness of intervention programmes. ${ }^{13}$ Since Sargent proposed the vertical jump as a physical performance test for men in 1921, considerable change has taken place, both in our thinking about physical performance and physical fitness, and about its measurement. ${ }^{14}$ Today, it is generally believed that a battery of tests should be used to assess an individual's physical 
Table 4: Correlations found

\begin{tabular}{|c|c|c|c|c|c|}
\hline & & $\begin{array}{l}\text { Laboratory-assessed } \\
\text { HRF index }\end{array}$ & $\begin{array}{c}\text { Direct } \mathrm{VO}_{2} \max (\mathrm{ml} / \\
\mathrm{kg} / \text { minute) }\end{array}$ & Kasari index & $\begin{array}{l}\text { Self-assessed HRF } \\
\text { index }\end{array}$ \\
\hline \multirow{2}{*}{$\begin{array}{l}\text { Direct } \mathrm{VO}_{2} \max (\mathrm{ml} / \mathrm{kg} / \\
\text { minute) }\end{array}$} & $\rho$ & $0.512^{* *}$ & & & \\
\hline & $p$ & $(0.005)$ & & & \\
\hline \multirow[t]{2}{*}{ Kasari index } & $\rho$ & 0.133 & 0.072 & & \\
\hline & $p$ & $(0.151)$ & $(0.731)$ & & \\
\hline Self-assessed HRF index & $p$ & $(<0.001)$ & $(0.068)$ & $(0.137)$ & \\
\hline \multirow{2}{*}{$\begin{array}{l}\mathrm{VO}_{2} \text { max indirect }(\mathrm{ml} / \mathrm{kg} / \\
\text { minute), calculated from } \\
\text { the Cooper } 12 \text { minute } \\
\text { walk/run test }\end{array}$} & $\rho$ & $0.588^{* *}$ & $0.794^{* *}$ & $0.278^{* *}$ & $0.454^{* *}$ \\
\hline & $p$ & $(<0.001)$ & $(<0.001)$ & $(0.002)$ & $(<0.001)$ \\
\hline
\end{tabular}

HRF: health-related fitness, $\rho$ : rho, $\mathrm{VO}_{2}$ max: maximum oxygen uptake, $p$ : statistical significance of the correlation

**: Highly significant correlations were found between:

- The participants' self-assessed HRF index and the HRF index determined by sports scientists

- The laboratory-assessed HRF index and the $\mathrm{VO}_{2}$ max determined in a laboratory

- $\quad$ An activity-recall questionnaire (Kasari) and the $\mathrm{VO}_{2}$ max

- Participants' self-assessed HRF index and the direct and indirect $\mathrm{VO}_{2}$ max

fitness. The HRF index was developed to measure the five major health-related components of physical fitness, namely cardiorespiratory fitness, muscular strength, muscular endurance, body composition and flexibility. Therefore, the intention of the index is to provide an indication of an individual's current overall physical status, and also to determine if there are specific areas that are lacking or below normal values, which would therefore require attention by means of appropriate intervention. The HRF index makes use of field tests that can be conducted with minimal equipment and expertise. Thus, individuals can regularly and easily assess themselves at home. Each test included in the index is a validated assessment of each of the five health-related components of physical fitness.

The correlation between the HRF index assessed by biokineticists and sport scientists from the Institute for Sport Research, University of Pretoria, and that of the participants themselves, was substantial $(\rho=0.696, p<0.001)$. The correlation strength suggests that the HRF index can be completed with reasonable accuracy by individuals. Therefore, it does not have to be conducted by specially trained individuals, such as sport scientists or biokineticists. Thus, the HRF test can be used to monitor progress or change in physical fitness without much expense or cost. The Kasari questionnaire correlated only weakly, but significantly, with the indirect $\mathrm{VO}_{2} \max (\rho=0.278, p=0.002)$, and not at all with the direct $\mathrm{VO}_{2} \max (\rho=0.072, p=0.731)$. Thus, the Kasari physical activity questionnaire was not a good indicator of participants' fitness and is therefore not a reliable substitute for physical fitness testing.

Cardiorespiratory endurance is one of the most important components of physical fitness. This refers to the ability to perform dynamic exercise involving large muscle groups at moderate to high intensity for prolonged periods. ${ }^{15}$ The $\mathrm{VO}_{2} \max$ is widely accepted as the measure of cardiorespiratory fitness. ${ }^{16} \mathrm{~A}$ test (indirect) that estimates the $\mathrm{VO}_{2}$ max was included in the HRF index, i.e. the Cooper 12 minute run/walk test. There was an almost perfect $(\rho=0.83)$ correlation between the directly measured $\mathrm{VO}_{2} \max (\mathrm{ml} / \mathrm{kg} /$ minute) on the treadmill and the indirectly estimated $\mathrm{VO}_{2}$ max by means of the Cooper 12 minute run/walk test. Previous studies have found the correlation between distance run tests and the $\mathrm{VO}_{2} \max$ to vary from $\rho=0.27-0.90$, depending on the participants, sample size and testing procedure. ${ }^{17-19}$

The self-assessed HRF index at baseline indicated a fair correlation ( $\rho=0.387$ ) with the direct measured $\mathrm{VO}_{2}$ max and a moderate to strong highly significant correlation $(\rho=0.454),(p<0.001)$ with the indirect $\mathrm{VO}_{2}$ max. This validates the potential of this instrument for use as a noninvasive battery of tests to easily evaluate or selfassess health-related fitness.

This difference in association strength ( $\rho$ between 0.5 and 0.6 ), compared to the previously mentioned $\rho=0.83$, can be explained by the fact that the $\mathrm{VO}_{2}$ max or cardiorespiratory fitness was only one component of the HRF index. Furthermore, the correlation was higher between the HRF index and the indirect assessment of the $\mathrm{VO}_{2}$ max since the indirect measure formed part of the HRF index battery of tests.

A study limitation included the fact that more women than men volunteered to take part in the study. Also, the fact that only keen and enthusiastic participants took part could be a confounding factor. It is recommended that the study protocol should be repeated in overweight patients who need to exercise.

\section{Conclusion}

It was confirmed the Kasari physical activity questionnaire was not a good indicator of participants' fitness. However, there was a statistically highly significant correlation between the HRF index obtained by participants at home and by themselves, and the HRF index, measured by biokineticists and sport scientists in standardised conditions. These test results may indicate that the HRF index could be used at home by individuals to monitor progress or change in physical fitness without much expense or cost. The information obtained in this way could:

- Enable trainers and clinicians to advise clients on their level of fitness via the Internet.

- Lead to distant health assessment by specialists and clinicians.

- Enable epidemiological research via the Internet.

- Enable people from rural communities to self-assess their health-related fitness. 
Acknowledgements - The HRF index project was funded by Momentum Interactive.

\section{References}

1. Heyward $\mathrm{VH}$. Advanced fitness assessment and exercise prescription. 6th ed.. Champaign, IL: Human Kinetics; 2010.

2. Grant S, Corbett K, Amjad AM, Wilson J, Aitchison T. A comparison of methods of predicting maximum oxygen uptake. Br J Sports Med. 1995;29(3):147-52. http://dx.doi.org/10.1136/bjsm.29.3.147

3. Bouchard C, Shepard RJ. Physical activity, fitness and health consensus statement. Champaign, IL: Human Kinetics; 1993.

4. Paterson DH, Govindasamy D, Vidmar M, Cunningham DA, Koval JJ. Longitudinal study of determinants of dependence in an elderly population. J Am Geriatr Soc. 2004;52(10):1632-8. http://dx.doi.org/10.1111/ jgs.2004.52.issue-10

5. Stringer WW. Cardiopulmonary exercise testing: current applications. Expert Rev Respir Med. 2010;4(2):179-88.

6. Prince SA, Adamo KB, Hamel ME, et al. A comparison of direct versus self-report measures for assessing physical activity in adults: a systematic review. Int J Behav Nutr Phys Act. 2008;5(1):1-24. http://dx.doi.org/10.1186/1479-5868-5-56

7. Cooper KR. A means of assessing maximal oxygen intake. JAMA. 1968;203(3):201-4. http://dx.doi.org/10.1001/jama.1968.031400300 33008

8. Sharkey BJ. Fitness and health. 4th ed. Champaign, IL: Human Kinetics; 1997.

9. Strydom GL, Kotze JP, Roux GF, et al. The physical activity profile of South African whites (men and women, 10-64 years) in Transvaal cities (VIGHOR-Study). S Afr J Res Sport Phys Educ Recreation. 1991;14(2): 65-76.
10. Van der Merwe S. Physical activity and psychological health in white males from Potchefstroom. Nort-West University; 1995. This is a dissertation published during 1995 by a student https://www.researchgate.net/publication/33938970_Fisieke_aktiwiteit_en_geestelike_welstand_van_blanke_mans_in_Potchefstroom_

11. Field A. Discovering statistics using SPSS. 2nd ed. London: Sage; 1994.

12. Landis JR, Koch GG. The measurement of observer agreement for categorical data. Biometrics 1977;33(1):159-74. http://dx.doi.org/10.23 $07 / 2529310$

13. Fox S, Dinur Y. Validity of Self-Assessment: A Field Evaluation. Pers Psychol. 1988;41(3):581-92. http://dx.doi.org/10.1111/peps.1988.41. issue-3

14. Sargent DA.The physical test of a man. Am Phys Edu Rev. 1921;26: 188-194.

15. Thompson WR, Gordon NF, Pescatello LS. ACSM's guidelines for exercise testing and prescription. 8th ed. Philadelphia, PA: Lippincott Williams \& Wilkins; 2010.

16. Heyward VH. Advanced fitness assessment and exercise prescription. 6th ed. Champaign, IL: Human Kinetics; 2010.

17. Zwiren LD, Freedson PS, Ward $A$, et al. Estimation of $\mathrm{VO}_{2}$ max: a comparative analysis of five exercise tests. Res Q Exercise Sport. 1991;62(1):73-8. http://dx.doi.org/10.1080/02701367.1991.10607521

18. Rikli RE, Petray $C$, Baumgartner TA. The reliability of distance run tests for children in grades K-4. Res Q Exercise Sport 1992;63(3):270-6. http://dx.doi.org/10.1080/02701367.1992.10608742

19. George JD, Vehrs PR, Allsen PE, Fellingham GW, Fisher AG. VO max estimation from a submaximal 1-mile track jog for fit college-age individuals. Med Sci Sports Exerc. 1993;25(3):401-6.

Received: 01-08-2013 Accepted: 03-10-2013 